\title{
Computer simulation of pressure sintering
}

\author{
N RAMAKRISHNAN, T BALAKRISHNA BHAT and \\ V S ARUNACHALAM* \\ Defence Metallurgical Research Laboratory, Hyderabad 500258 , India \\ *Scientific Adviser to Defence Minister, New Delhi 110011, India
}

\begin{abstract}
The densification process during pressure sintering has been analyzed using finite element analysis. This analysis uses an iterative solution algorithm. With this the densification process in complex geometries with complex boundary conditions can be analyzed and this technique is particularly suited for tackling material nonlinearity. Evolution of dense structures with gradual closure of pores is described for two typical geometries.
\end{abstract}

Keywords. Computer simulation; pressure sintering; finite element analysis; creep modulus

\section{Introduction}

Pressure sintering is a technique in which a powder aggregate is subjected to high pressures at high temperatures to achieve simultaneous compaction and sintering. Theoretical analysis of pressure sintering is greatly restricted due to the complexities involved in the geometries and the nonlinear material properties. Usually, powder aggregates are idealized to simple geometries (McKenzie 1948; Coble 1970; Kovalachenko and Samsonov 1961; Wilkinson and Ashby 1975; Notis and Krishnamachary 1975) disregarding the spatial and size distribution of pores or the interaction of pores. These problems can be reduced substantially by the use of numerical techniques and we have chosen finite element method (FEM) for analyzing pressure sintering with all its complexities, as it provides a simple and direct approach to handle complex geometries, boundary conditions and material behaviour (Ramakrishnan et al 1984).

\section{Simulation of powder aggregates}

Two types of two-dimensional configurations have been identified for simulating the powder aggregates: (i) randomly packed spheres of log normal size distribution (figure 3a) (ii) randomly distributed spherical pores of log normal size distribution in a homogenous matrix (figure 4a). To construct the first geometry, a hypothetical arrangement of circular discs of log normal size distribution placed in a rectangular box has been considered. The packing is done starting from the bottom row ensuring the mechanical stability of the arrangement. This geometry represents the initial stage of compaction of powder aggregates.

The second geometry is formed by randomly placing circular pores of log normal size distribution in a rectangular material matrix. This geometry depicts the final stage of sintering where pores become spherical due to surface tension and the material matrix 
becomes nearly continuous. These geometries are subdivided into a number of small rectangular and triangular elements for finite element analysis. As the stress varies rapidly near the pores, a finer discretization is performed there.

\section{Analysis of densification of powder aggregates}

Densification in pressure sintering takes place mainly through creep processes such as diffusion creep, dislocation creep and grain boundary sliding. These processes are driven by the local stresses. Therefore for the analysis of densification it is necessary to compute the stresses at various locations in the material matrix for the applied external pressure and the boundary conditions. These stresses are in turn used to compute the strains and the displacements for a given interval of time at the corresponding locations to obtain the new geometry. Again the new geometry is subjected to similar analysis and this process is continued in an iterative fashion until the porosity reaches the desired low value. The increments in time are decided in such a way that the change in geometry in any particular iteration is small.

\section{Finite element analysis (FEA) of creep deformation}

In FEA of creep deformation, the behaviour of each element is described in terms of force-displacement rate relationship. The governing system of equations for the whole geometry is established by the assembly of elemental behaviour, subject to compatability and dynamic equilibrium conditions at each node. These equations are solved to yield stresses and displacement rates at various nodes.

\subsection{Basic equations}

The force-displacement rate relation is given by

$$
\{F\}^{e}=[K]^{e}\{\dot{q}\}^{e}
$$

where $[K]^{e}$ represents the stiffness matrix, $\{\dot{q}\}$ the displacement rate vector and $[F]^{e}$ the nodal force vector. On assembly, these equations assume the following global form:

$$
\{F\}^{g}=[K]^{g}\{\dot{q}\}^{g}
$$

The stresses and strain rates in terms of displacement rates are given by the following equations:

$$
\begin{aligned}
& \{\dot{\epsilon}\}=[B]\{\dot{q}\}, \\
& \{\sigma\}=E_{c}^{e}[c]\{\dot{\varepsilon}\},
\end{aligned}
$$

where $[B]$ is the coefficient matrix, purely dependent on the geometry of the element and $[C]$ is given by (Donea 1978)

$$
[C]=\frac{1}{(1-2 \gamma)(1+\gamma)}\left[\begin{array}{ccc}
1-\gamma & \gamma & 0 \\
\gamma & 1 \frac{-\gamma}{0} & 0 \\
0 & 0 & 2(1+\gamma)
\end{array}\right]
$$


for plain strain condition where $\gamma$ is the Poisson's ratio. $E_{c}^{e}$ is the elemental creep modulus and for the constant strain elements we define it as

$$
E_{c}^{e}=\sigma_{*}^{e} / \dot{\varepsilon}_{*}^{e},
$$

where $\sigma_{*}$ and $\dot{\varepsilon}_{*}$ are effective stress and strain rates respectively. The stress-strain rate relationship is nonlinear and is generally represented in the form of power law

$$
\dot{\varepsilon}_{*}=A_{c} \sigma_{*}^{n}
$$

where $A_{c}$ and $n$ are creep constants which depend on the material and the temperature.

\subsection{Procedure for determining the stiffness matrix}

The minimum potential energy condition is used to establish the stiffness matrix. The potential energy can be represented as

$$
\pi=\left[\iiint\{\sigma\}^{T}\{\dot{\varepsilon}\} \mathrm{d} V-\iint\{P\}\{\dot{q}\} \mathrm{d} S\right] \Delta t,
$$

where $[P]$ represents forces per unit area and $\Delta t$ a small interval of time. Using (3) and (4), (7) can be modified to

$$
\pi=\left[\iiint E_{c}^{e}\{\dot{q}\}^{T}[B]^{T}[C][B]\{\dot{q}\} \mathrm{d} v-\iint\{P\}\{\dot{q}\} \mathrm{d} S\right] \Delta t .
$$

For dynamic equilibrium $\delta \pi / \delta \dot{q}=0$. Assuming the displacement rates to be constant in a sufficiently small interval of time, the above criterion can be shown to give,

$$
F=2 E_{c}^{e}[B]^{T}[C][B]\{\dot{q}\} h S_{e},
$$

where $h$ and $S_{e}$ are the thickness and the area of the element respectively, within which strain is assumed constant. Hence stiffness matrix for creep is given by

$$
[K]^{e}=2 h S_{e}[B]^{T}[C][B] E_{c}^{e} .
$$

\subsection{Procedure for analyzing densification}

To start with, the elemental creep modulus of all the elements are assumed to be identical and an arbitrary $E_{c}^{e}$ is assigned to all the elements. The displacement rates $\{\dot{q}\}$ and nodal forces $[F]$ are obtained by solving (9) for the given boundary conditions. $\{\sigma\}$ and $\{\dot{\varepsilon}\}$ are then computed using (3) and (4). These stresses do not correspond to actual stresses and the actual stresses can be calculated only by an iterative procedure that gives a convergent, compatible solution. Effective stresses $\sigma_{*}$ and effective strain rates $\dot{\varepsilon}_{*}$ are calculated using the equations

$$
\sigma_{*}^{2}=\left(\sigma_{1}-\sigma_{2}\right)^{2}+\left(\sigma_{2}-\sigma_{3}\right)^{2}+\left(\sigma_{3}-\sigma_{1}\right)^{2},
$$

where $\sigma_{1}, \sigma_{2}, \sigma_{3}$ are the principal stresses

and $\quad \dot{\varepsilon}_{*}=A \sigma_{*}^{n}$.

The creep moduli for the subsequent iteration is computed by the equation

$$
E_{c}^{e}=(1 / A)^{1 / n}\left(\dot{\varepsilon}_{*}\right)^{1 / n-1}
$$

Using these creep moduli, new displacement rates are obtained. These calculations are continued until the stresses converge in each element. After the convergence, the nodal 


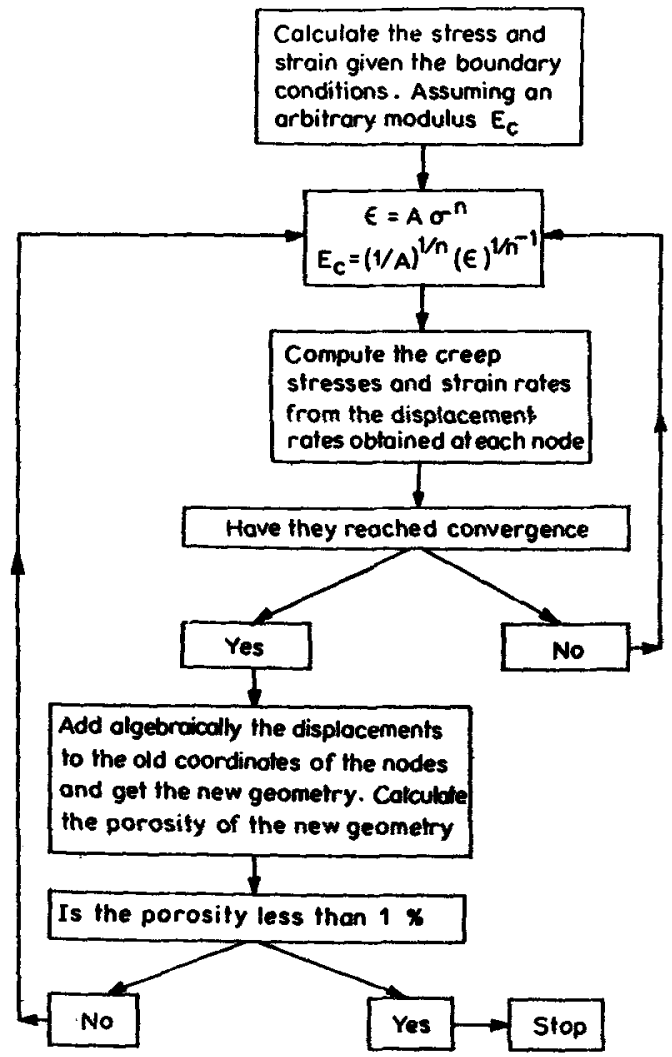

Figure 1. Flow chart for computer simulation of pressure sintering.

co-ordinates are updated using the displacements at each node and the procedure is repeated for the new porosity. In figure 1 , the whole procedure is given in the form of a flow chart.

\subsection{Boundary conditions}

Either force boundary conditions or displacement rate boundary conditions can be thought of for this analysis; the first one appears apparently simple and more relevant as what we know in practice is only the external pressure. But if we impose force boundary conditions, the displacement rates computed for all the boundary nodes may not be perfectly same as we are not dealing with a perfectly random system. The distortions in the geometry introduced by this becomes increasingly pronounced in subsequent iterations. To overcome this problem the following procedure is adopted.

Unit displacement rate is imposed on the boundary nodes ensuring that there are no contradictions, in representing the actual situation. For example in isostatic pressing as in figure $2 a$, the boundary conditions given are,

for

$$
x=l,\left\{\dot{q}_{x}\right\}=-1 ; \text { for } y=l,\left\{\dot{q}_{y}\right\}=-1 ;
$$

for

$$
x=0, y=0,\left\{\dot{q}_{x}\right\}=\left\{\dot{q}_{y}\right\}=0 \text {. }
$$




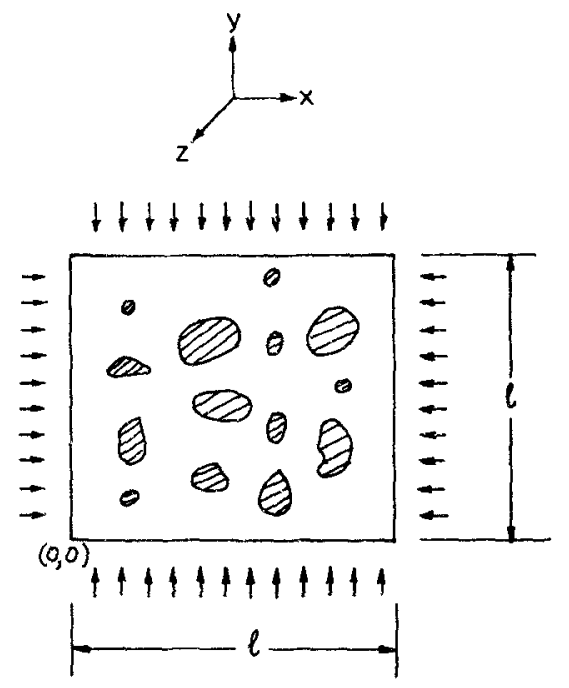

(a)

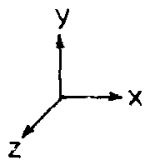

$p$

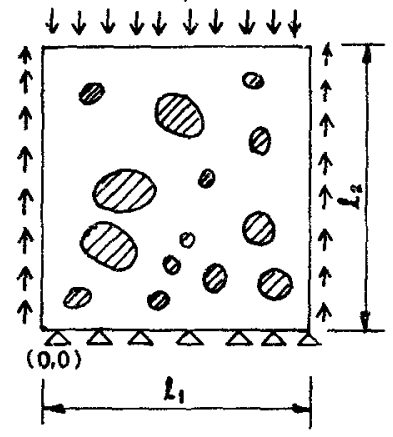

(b)

Figure 2. Boundary conditions for (a) isostatic pressing (b) uniaxial pressing with boundary wall friction.

Using (2) we can obtain the forces at various nodes needed to produce these displacement rates. These forces can be used to calculate the dummy pressure $P^{\prime}$ and if $P$ is the actual pressure, we normalize all the calculated values using the scaling factor

$$
\lambda=P^{\prime} / P \text {. }
$$

The relationship between dummy and actual displacement rates is given by

$$
\left\{\dot{q}^{\prime}\right\}=\lambda\{\dot{q}\} .
$$

The actual boundary conditions used in this analysis for (i) isostatic pressing (ii) uniaxial pressing are shown in figures $2 \mathrm{a}, \mathrm{b}$ respectively.

\subsection{Updating the geometry}

Once the convergence of stresses is established for any particular porosity, the displacements are calculated at each node for a given time increment and these are algebrically added to the original coordinates of the nodes to get the new geometry. To have a control over the change in the geometry with densification, the incremental boundary displacements are fixed instead of fixing the incremental time intervals and for each step, time increment is actually calculated.

It is often necessary to refine and regenerate the mesh at regular intervals. Otherwise the elements may start overlapping when a particular pore is completely closed. If two nodes come very close, then one of the nodal data is removed from the nodal information directory and correspondingly element directory is modified. If the segments joining the nodes surrounding the pores intersect, a new node is created at the 
point of intersection and the nodal and elemental directories are appropriately altered. The resulting node number sequence may increase the bandwidth of the stiffness matrix. Therefore the region of interest is scanned and the nodal and the elemental directories are modified in the whole region to bring down the bandwidth.

\section{Results of the computer simulation}

\subsection{Evolution of pore geometries}

During pressure sintering, the geometry of the powder compact undergoes a variation with time. Figure 3 is a typical sequence depicting this variation in a powder aggregate. It may be seen that the smaller particles and the boundary regions of coarser particles only get deformed significantly and not the interior of a large particle. Figure 4 represents isostatic pressing and figure 5 represents uniaxial pressing with die wall friction taken into account. Figure 5 particularly brings out the density variation at various locations similar to earlier experimental curves obtained for uniaxial pressing (Jones 1960).

\subsection{Pore size variation}

The decrease in pore sizes with densification is depicted in figure 6. It is very clearly seen from the figure that the distribution of pore sizes narrows down with densification. Also
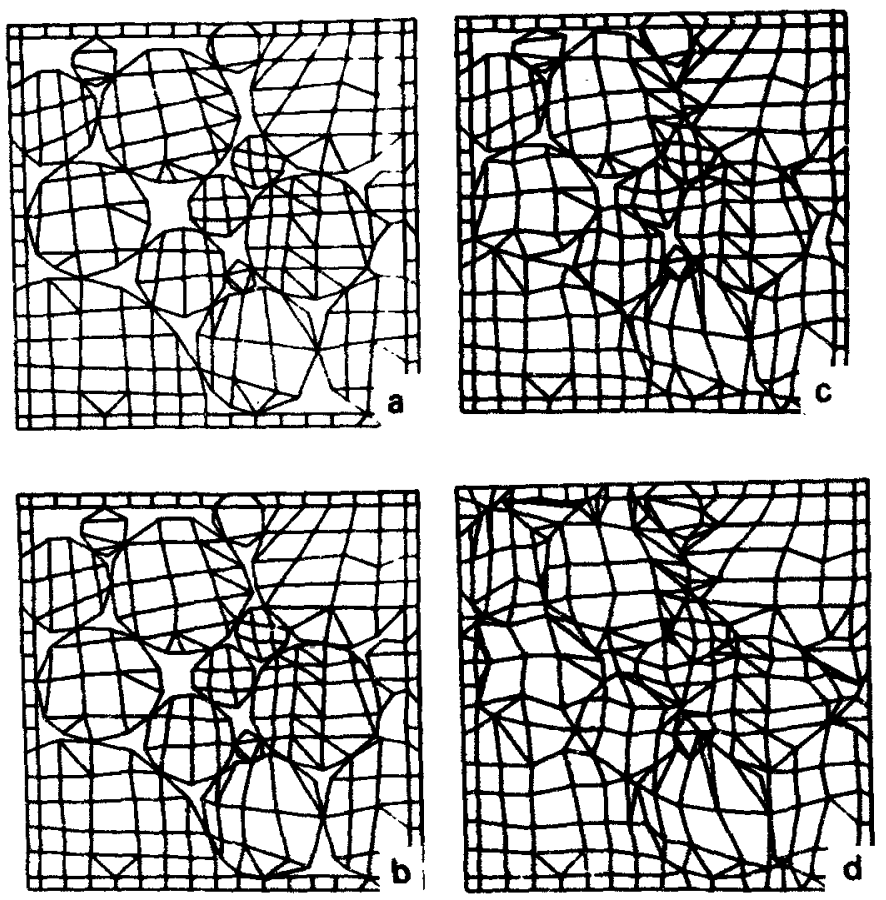

Figure 3. Sequence of closure in a powder compact during isostatic pressing. 

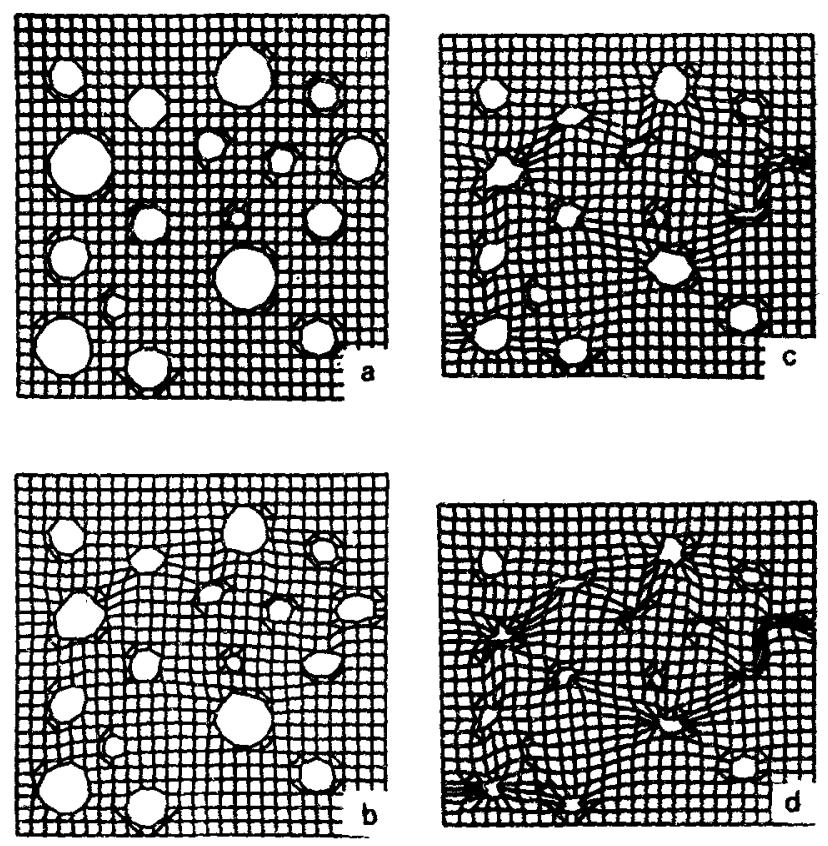

Figure 4. Sequence of pore closure in a compact with initially rounded pores during isostatic pressing.
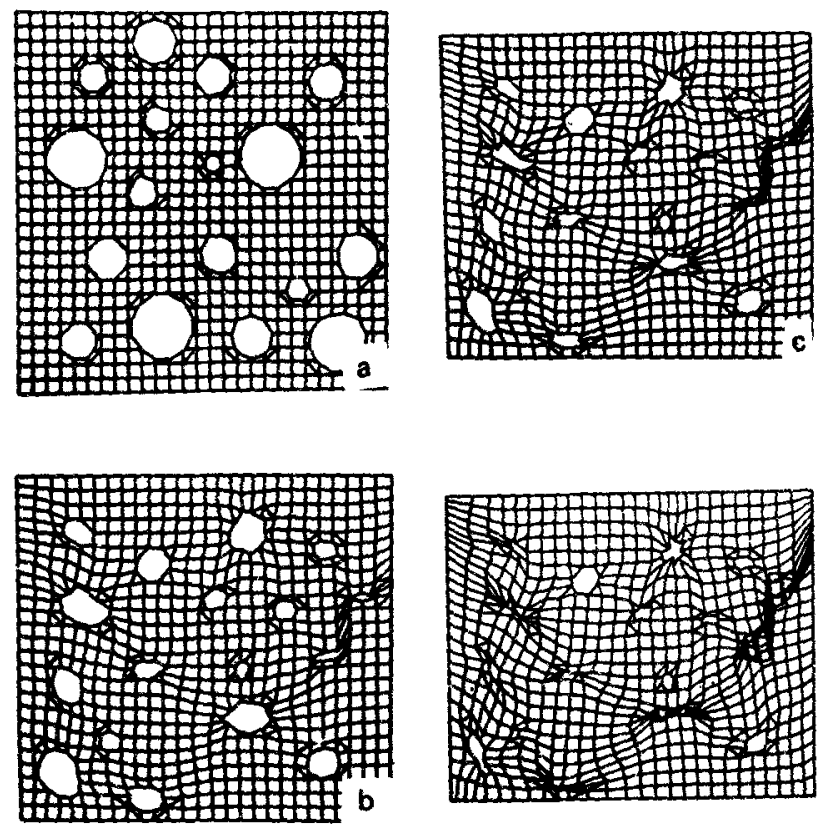

Figure 5. Sequence of pore closure in a compact with initially rounded pores during uniaxial pressing with boundary wall friction. 


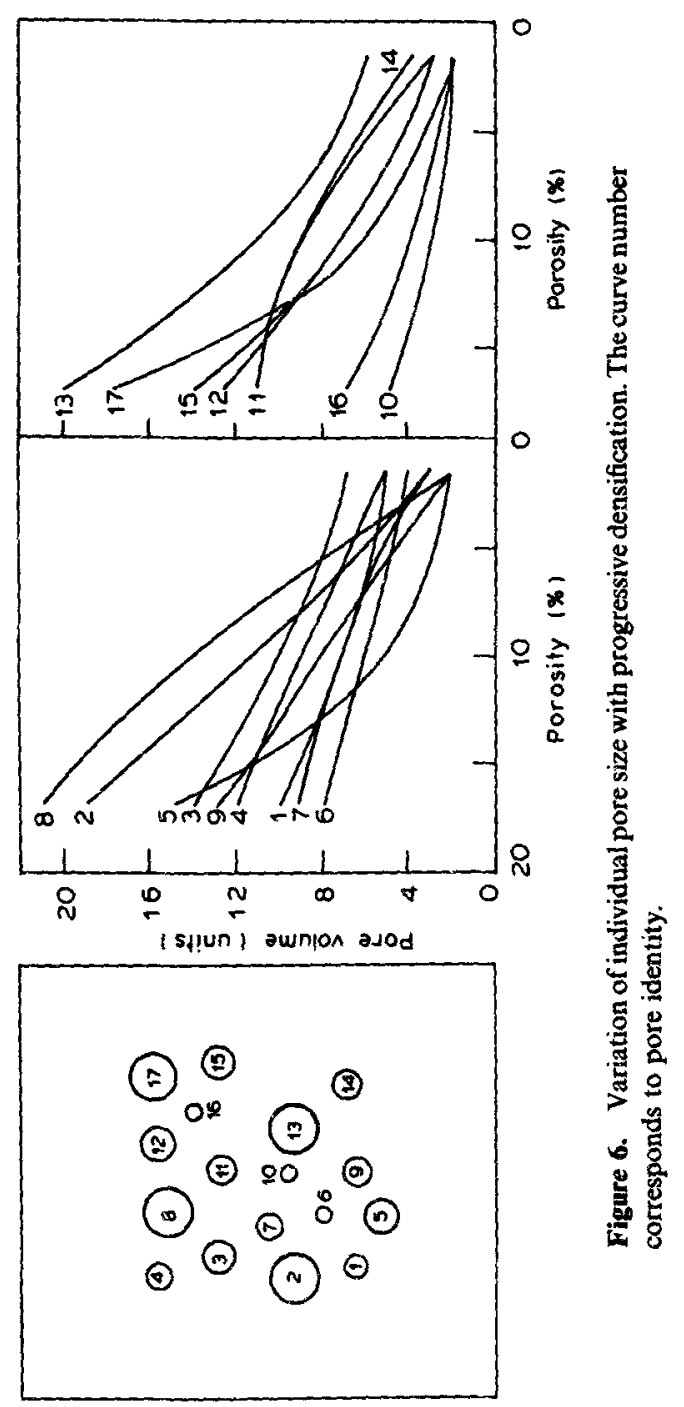


curves crossing each other suggests that the closure rate not only depends on its size but also on its neighbours.

\section{Conclusion}

Finite element analysis can be used to analyze complex geometries of porous aggregates sintering under pressure. Even nonlinear material flow behaviour can be taken into account using a concept of creep modulus to provide a globally convergent solution. The technique applied to a compact containing a set of pores indicates that the closure of any given pore is governed not only by its own size but also its neighbourhood.

\section{References}

Coble R L 1970 J. Appl. Phys. 414798

Donea $\mathrm{J} 1978$ Creep in engineering materials and structures (eds) G Barnasconi and G Piati (London: Applied Science Publishers) p. 34

Jones W D 1960 Fundamental principles of powder metallurgy (Leeds; Arnold) p. 299

Kovalachenko M/S and Samsonov G V 1961 Poroshkov Metall. 13

McKenzie J K 1948 Proc. R. Soc. (London) B62 833

Notis M R and Krishnamachary V 1975 Sintering and catalysis (eds) G C Kuczinsky (New York; Plenum Press) p. 493

Ramakrishnan N, Balakrishna Bhat T and Arunachalam V S 1984 Acta Metall. 32357

Wilkinson D S and Ashby M F 1975 Acta Metall. 231277 